\section{Addressing the demand for termination of pregnancy services in district health facilities in Johannesburg}

To the Editor: The Choice of Termination of Pregnancy (CTOP) Act instituted safe, effective, affordable and acceptable methods of fertility regulation for women. Universal access to reproductive health services is available through the district health services. ${ }^{1}$

By 2001, there had been a $91 \%$ decline in maternal mortality from unsafe abortions in South Africa (SA) as a result of the CTOP Act. ${ }^{2}$ However, despite the availability of free reproductive health services, few women are utilising family planning services in SA. ${ }^{3}$ Recent studies have reported that unsafe abortions are on the increase. ${ }^{4-6}$

We wished to determine the number of terminations of pregnancy (TOPs) requested and the number of TOPs performed from January 2008 to December 2009 in the Johannesburg Metropolitan District (JM). We conducted a descriptive cross-sectional study including TOP data from district health information systems in the JM. All health facilities offering TOP services at the district level were included.

The analyses showed that a total of 14683 and 16031 women requested TOPs in 2008 and 2009 respectively; these figures might have included women in their second trimester, at district facilities. A third of requests were performed, with 4921 and 5338 first-trimester TOPs performed in 2008 and 2009 respectively (Fig. 1). In 2008, a total of 6 clinics offered TOP services in the JM. The majority (68\%) of the first-trimester TOPs were performed by 2 facilities. Two clinics which collectively had performed $15 \%$ of the total number of TOPs in 2008 , ceased offering TOP services in 2009; only a new clinic initiated TOP services in 2009.

Although the number of TOP requests increased from 2008 to 2009 , the facilities offering first-trimester TOP services declined. In addition, the number of first-trimester procedures performed was far less than the number of requests received. A major concern is that if the demand for TOPs remains unmet in the public sector, the incidence of unsafe abortions may continue to rise further.

\section{J F Mendes \\ D Basu}

School of Public Health

Charlotte Maxeke Johannesburg Academic Hospital, and

University of the Witwatersrand

Johannesburg

jacqui.mendes@wits.ac.za

\section{J K Basu}

Department of Obstetrics and Gynaecology

Charlotte Maxeke Johannesburg Academic Hospital, and

University of Witwatersrand

Johannesburg

1. Choice of Termination of Pregnancy Act No. 92 of 1996. Pretoria: Department of Health, 1996.

2. Jewkes R, Rees H. Dramatic decline in abortion mortality due to choice of Termination of Pregnancy Act. S Afr Med J 2005; 95: 250

3. MacPhail C, Pettifor AE, Pascoe S, Rees H. Contraception use and pregnancy among 15 - 24 years old South African women: a nationally representative crosssectional survey. BMC Med 2007; 28: 31

4. Meel B, Kaswa RP. The impact of Choice of Termination of Pregnancy Act of 1996 (Act 92 of 1996) on criminal abortions in the Mthatha area of South Africa. African Journal of Primary Health Care and Family Medicine 2009; 1: 79-81.

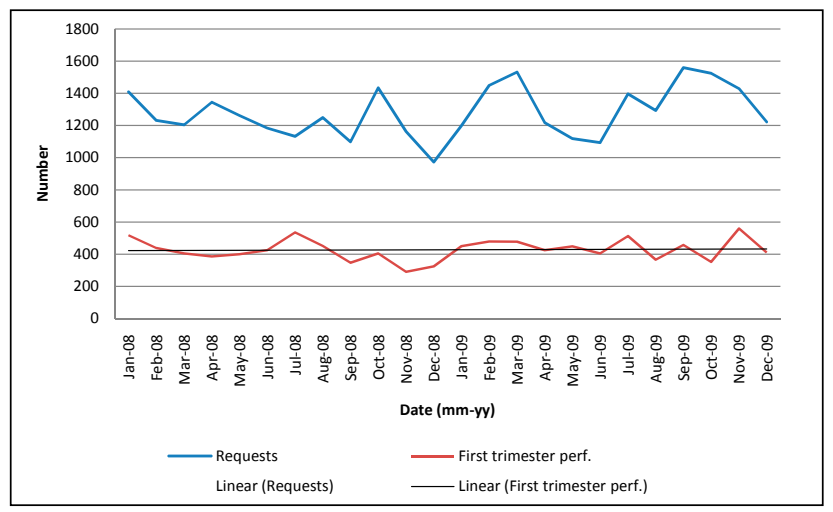

Fig. 1. Distribution of number of TOP requests and first trimester TOP procedures performed per month from January 2008 to December 2009 in the Johannesburg Metropolitan District.

5. Basu JK. Legal unsafe abortion in South Africa. Conference proceeding XIX FIGO World Congress of Gynaecology and Obstetrics, Cape Town, South Africa, 4 - 9 October 2009.

6. Morroni C, Moodley J. Characteristics of clients seeking first and second trimester termination of pregnancy in public health facilities in Cape Town. S Afr Med J 2006; 96: 574

\section{Medical indemnity regulations: MPS maintains commitment to South Africa}

To the Editor: I want to reassure members of the Medical Protection Society and readers of the SAMJ that the surprise new government regulations on indemnity will not diminish our longstanding and strong commitment to the South African medical and dental professions.

We had no warning of the new regulations and we know their publication has caused a great deal of uncertainty, but the regulations will not affect MPS members until the end of December and in the meantime we are committed to doing everything we can to find a long-term solution.

The MPS has operated in South Africa for over 50 years and we want to be here for another 50 . We have 25000 members in South Africa, and each of them is important to us. As a mutual, our ethos, when looking at whether to offer our services and support in a country, is to ask the question: does the profession want us? We have been overwhelmed by the support we have received from our members over the past week, and this has galvanised our search for a solution that will allow the MPS to continue to indemnify and support doctors and dentists in South Africa. We are also encouraged by the response of government agencies and regulators who have voiced a strong desire for us to remain.

We do not dispute the principle behind the regulations; we agree that medical professionals should have compulsory professional indemnity so that they and their patients are protected if something avoidable goes wrong. We also support the requirement that indemnifiers should be capable of regulation. Our objection - our strong objection - is the preclusion of the indemnity that the MPS provides, which is widely regarded as the best available. Although it is discretionary, it allows us the flexibility to provide help and support in circumstances where a claim may otherwise be rejected by an insurer. In our long history there has been no case of the MPS declining to meet a proven claim of negligence that has resulted in a patient being left uncompensated. The occurrence-based nature 